\title{
Physical Activity Status and Body Image Perception of Adolescent Females in A Slum In Kolkata, India.
}

\author{
Dr Sunetra Kaviraj ${ }^{1}$, Dr. Abhik Sinha ${ }^{2}$, Dr. Nabanita Chakraborty ${ }^{1}$, \\ Dr. Himanish Roy ${ }^{1}$, Dr. Ronjoy Majumdar ${ }^{1}$, Dr. Murari Mohan Mondal ${ }^{1}$ \\ ${ }^{I}$ Post Graduate Trainee, Dept of Community Medicine, R. G Kar Medical College, Kolkata \\ 2 \\ Assistant Professor, Dept of Community Medicine, $R$ G Kar Medical College, Kolkata
}

\begin{abstract}
Introduction: Physical activity is defined as any bodily movement produced by skeletal muscles that requires energy expenditure. Physical inactivity has been identified as the fourth leading risk factor for global mortality causing an estimated 3.2 million deaths globally.

Objectives: To find the physical activity status of the adolescent girls and to know their body image perception. Materials And Methods: The study was conducted at the Bagbazar Slum of Urban Field Practice Area of R.G.Kar Medical College. A total of 107 girls from 10 to 19 years of age residing in this slum participated in this study. A pre-designed, pre-tested questionnaire was used for data collection. For determining physical activity status Global Physical Activity Questionnaire (GPAQ) was used and the results were compared with the WHO guidelines for physical activity for adolescents. METs (Metabolic Equivalents) are used for the analysis of GPAQ data. MET is the ratio of a person's working metabolic rate relative to the resting metabolic rate. One MET is defined as the energy cost of sitting quietly and is equivalent to a caloric consumption of $1 \mathrm{kcal} / \mathrm{kg} / \mathrm{hour}$. Therefore, when calculating a person's overall energy expenditure using GPAQ data, 4 METs get assigned to the time spent in moderate activities, and 8 METs to the time spent in vigorous activities.

Results: Only 14.28\% of girls in the age group 10 to 17 years and $67.56 \%$ of girls above 17 years had physical activity above recommended guidelines. 57\% of girls had no recreation involving physical activity of any kind. 47.7\% of girls were not satisfied with their body image.

Conclusion: Physical activity should be promoted among adolescent girls to prevent occurrence of NCD and lead a healthy life.
\end{abstract}

Key Words: Adolescent, Perception, Body Image, Physical Activity

\section{Introduction:}

Physical activity is any bodily movement by skeletal muscles that requires energy expenditure. Physical inactivity is the fourth leading risk factor for mortality causing an estimated 3.2 million deaths globally ${ }^{1}$. Physical activity is one of the major lifestyle-related health determinants. Evidence suggests that from an early age, differences in gender-based attitudes towards and opportunities for sports and physical activities can have a significant influence on children's participation ${ }^{2}$. This may, in turn, affect later involvement in physically active lifestyles, and the social and health benefits that may result for them. Hence knowing the physical activity status of adolescent girls would be an important step towards determining the risk of them developing non-communicable diseases in the future.

WHO developed the "Global Recommendations on Physical Activity for Health" with the overall aim of providing national and regional level policy makers with guidance on the dose-response relationship between the frequency, duration, intensity, type and total amount of physical activity needed for the prevention of noncommunicable diseases $(\mathrm{NCD})^{3}$.

Adolescence is a period of not only physical but various emotional changes as well. Girls in this age group have an overbearing desire to look beautiful, leading them to feelings of wrong body image perceptions and hence feelings of insecurity and low self esteem. This study tries to identify the self image perception among the adolescent girls vis-a-vis their true body mass indices.

Objectives: To find the physical activity status of adolescent girls and to know their body image perception.

\section{Materials And Methods:}

The cross-sectional study was conducted at Bagbazar Slum, the Urban Field Practice Area of Department of Community Medicine, R.G.Kar Medical College and Hospital from April 2012 to May 2012. All girls from 10 to 19 years of age residing in this slum who gave their consent to participate in this study. A pre-designed, pre-tested schedule was used for data collection. 
For determining physical activity status Global Physical Activity Questionnaire (GPAQ) ${ }^{4}$ was used and the results were compared to WHO guidelines for physical activity for adolescents. METs (Metabolic Equivalents) are used for analysis of GPAQ data. MET is the ratio of a person's working metabolic rate relative to the resting metabolic rate ${ }^{4}$. One MET is the energy cost of sitting quietly and is equivalent to a caloric consumption of $1 \mathrm{kcal} / \mathrm{kg} /$ hour. Therefore, when calculating a person's overall energy expenditure using GPAQ data, 4 METs get assigned to time spent in moderate activities and 8 METs to time spent in vigorous activities.

\section{W.H.O Recommendations ${ }^{3}$ :}

\section{For 5 yrs to 17 yrs old:}

1.Children and youth aged 5-17 should accumulate at least 60 minutes of moderate- to vigorousintensity physical activity daily.

2. Amounts of physical activity greater than 60 minutes provide additional health benefits.

3. Most of the daily physical activity should be aerobic. Vigorous-intensity activities should be incorporated, including those that strengthen muscle and bone, at least 3 times per week.

\section{For 18yrs to 65 yrs old:}

1. Adults aged 18-64 should do at least 150 minutes of moderate-intensity aerobic physical activity throughout the week or do at least 75 minutes of vigorous-intensity aerobic physical activity throughout the week or an equivalent combination of moderate- and vigorous-intensity activity.

2. Aerobic activity should be performed in bouts of at least 10 minutes duration.

3. For additional health benefits, adults should increase their moderate-intensity aerobic physical activity to 300 minutes per week, or engage in 150 minutes of vigorous-intensity aerobic physical activity per week, or an equivalent combination of moderate- and vigorous-intensity activity.

4. Muscle-strengthening activities should be done involving major muscle groups on 2 or more days a week.

Height (to the nearest $0.1 \mathrm{~cm}$ ) and weight (to the nearest $0.5 \mathrm{~kg}$ ) was measured once with the subjects wearing light clothing and no shoes using standard technique and tools.

\section{Results:}

Mean age of the study population was 15.2 years $(S . D=3.134) .28 \%$ of the girls had education upto middle school.

Mean physical activity of the girls was 1280.93 METs ( $S . D=784.15) .85 .72 \%$ of the girls in the age group 10 to 17 years had physical activity below the recommended levels (Figure 1). As high as $57 \%$ of girls had no recreation involving physical activity of any kind.

$52.3 \%$ of the girls were satisfied with their body image. $34 \%$ of the girls thought they were fat, whereas $27 \%$ thought they were neither thin nor fat (Figure 2).

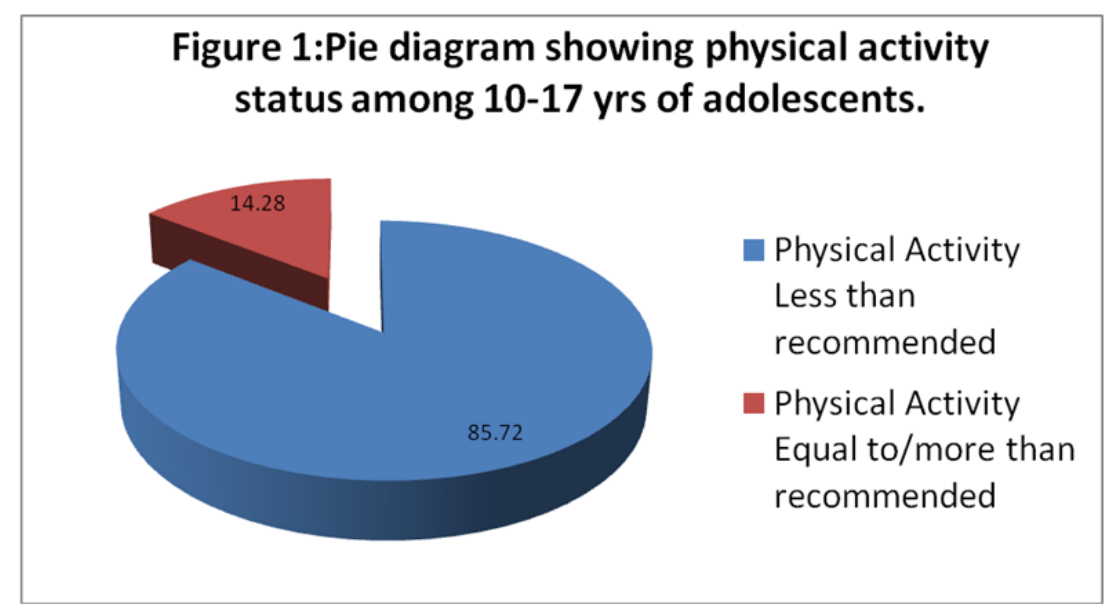




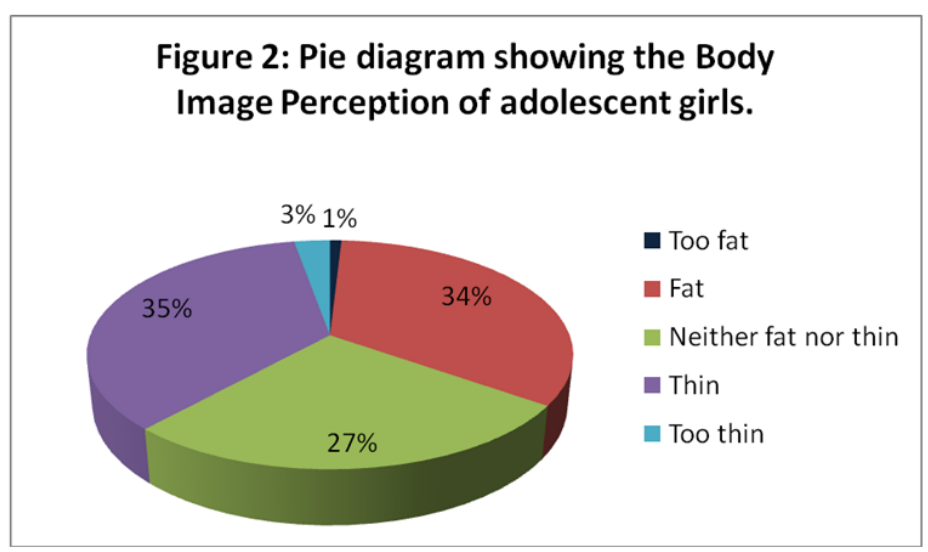

Table 1: Distribution of adolescent girls according to their body image perception and nutritional status. $(\mathrm{N}=107)$.

\begin{tabular}{|l|l|l|l|l|}
\hline Body Image Perception & Undernutrition & Normal nutrition & Overweight and Obese & Total \\
\hline Fat and Too Fat & $0 \%$ & $20.56 \%$ & $13.08 \%$ & $33.64 \%$ \\
\hline Neither Fat nor Thin & $0.93 \%$ & $25.23 \%$ & $0.93 \%$ & $27.10 \%$ \\
\hline Thin and Too thin & $11.21 \%$ & $27.10 \%$ & $0 \%$ & $38.31 \%$ \\
\hline Total & $12.14 \%$ & $72.89 \%$ & $14.01 \%$ & $100 \%$ \\
\hline
\end{tabular}

Table 1 . shows that $20.56 \%$ of the girls in normal nutritional status considered themselves fat. Also, $49.52 \%$ of the girls had body image perception conforming to their nutritional status.

Table 2: Distribution of adolescent girls according to physical activity and nutritional status. ( $\mathrm{N}=107)$.

\begin{tabular}{|l|l|l|l|l|}
\hline Activity & Undernutrition & Normal Nutrition & Overweight and Obese & Total \\
\hline Sedentary & $8.41 \%$ & $47.66 \%$ & $6.54 \%$ & $62.61 \%$ \\
\hline Active & $3.73 \%$ & $25.23 \%$ & $8.41 \%$ & $37.39 \%$ \\
\hline Total & $12.41 \%$ & $72.89 \%$ & $14.95 \%$ & 100 \\
\hline
\end{tabular}

Only $25.23 \%$ of the girls in normal nutritional status were physically active as per the WHO guidelines.

\section{Discussion:}

In a study conducted by Swati Dixit et al among female adolescents $73.4 \%$ of the girls were satisfied with their body image whereas $32.8 \%$ had overestimated their body weight. This is close to the results of this study where $20.56 \%$ of the girls in normal nutritional status considered themselves fat. Another study showed how $27.4 \%$ of the adolescent girls were of normal nutritional status and $86 \%$ of the girls desired to be slim ${ }^{6}$.

A study conducted on rural adolescents of West Bengal showed that $22 \%$ of the subjects were not sufficiently physically active ${ }^{7}$. But our study showed $67.6 \%$ of the subjects were not sufficiently active. This could be due to the urban location being the study setting of our study.

Also the study conducted in rural West Bengal among adolescents showed tha older giels spent their leisure time by doing sedentary activities ${ }^{7}$. This study showed similar results where $57 \%$ of the girls had no recreation involving physical activity of any kind. This could be due to lack of parental or social support for games.

\section{Conclusion:}

There is inadequate physical activity among the adolescent girls of Bagbazar slum. There should be parental and social support for physical activity and community based recreational opportunities.

National guidelines or recommendations on physical activity for the general population are needed to inform the population on the frequency, duration, intensity, types and total amount of physical activity necessary for health. However, increasing levels of physical activity in the population demands a population-based, multisectoral, multidisciplinary, and culturally relevant approach.

National policies and plans on physical activity should comprise multiple strategies aimed at supporting the individual and creating supportive environments for physical activity to take place.

Correct knowledge regarding healthy eating, exercise and body image should be imparted to all adolescents at school. Health education should be given to the adolescents regarding maintaining the proper nutritional status so that they can be at less risk for developing non-communicable diseases in the future. 


\section{References:}

[1]. Cardiovascular disease, obtained

from http://www.world-heartfederation.org/fileadmin/user_upload/documents/Fact_sheets/2012/Press Backgrounder April2012RiskFactors.pdf , last accessed on 27.06.2013.

[2]. Girls' participation in physical activities and sports: benefits, patterns, influences and ways forward, obtained from http://www.icsspe.org/sites/default/files/Girls.pdf, last accessed on 27.06.2013.

[3]. Global recommendations on physical activity for health, obtained http://www.who.int/dietphysicalactivity/factsheet_recommendations/en/index.html, last accessed on 24.06.2013.

[4]. Global Physical Activity Questionnaire, obtained from http://www.who.int/chp/steps/resources/GPAQ_Analysis_Guide.pdf, last accessed on 24.06.2013.

[5]. Swati Dixit, GG Agarwal, JV Singh, Surya Kant, Neelam Singh. A Study on Consciousness of Adolescent Girls About Their Body Image. Indian J Community Med. 2011 Jul-Sep; 36(3): 197-202.

[6]. Latha KS, Hegde S, Bhat, Sharma P, Rai P. Body image, self-esteem and depression in female adolescent college students. J Indian Assoc Child Adolesc Ment Health. 2006;2:78-84.

[7]. Sima Roy, Aparajita Dasgupta. Physical Activity Pattern Among the Adolescents of a Rural Community in West Bengal. Indian J Community Med. 2009 October; 34(4): 366-367. 